\title{
A novel familial mutation associated with Treacher Collins syndrome: A case report
}

\author{
ELENA PAPAGEORGIOU ${ }^{1}$, IOANNIS PAPOULIDIS ${ }^{1}$, APOSTOLOS ZAVLANOS $^{2}$, \\ EVAGGELOS PAPANIKOLAOU ${ }^{3}$, EMMANOUIL MANOLAKOS ${ }^{1}$ and STILIANI FIDANI ${ }^{4}$ \\ ${ }^{1}$ Access to Genome, Clinical Laboratory Genetics, 55134 Thessaloniki; \\ ${ }^{2} 1$ st Department of Obstetrics and Gynecology, Papageorgiou Hospital, 56403 Thessaloniki; \\ ${ }^{3} 3$ rd Department of Obstetrics and Gynecology, Ippokratio Hospital, Aristotle University of Thessaloniki, 54642 Thessaloniki; \\ ${ }^{4}$ Department for Special Needs, Aristotle University of Thessaloniki Achepa Hospital, 54636 Thessaloniki, Greece
}

Received February 7, 2019; Accepted October 21, 2019

DOI: $10.3892 /$ br.2020.1284

\begin{abstract}
Treacher Collins syndrome (TCS) is a type of mandibulofacial dysostosis with incomplete penetrance and high intra- and interfamilial clinical heterogeneity, and it is associated with mutations of treacle ribosome biogenesis factor 1 (TCOF1), and RNA polymerase I and III subunit (POLR1)C and POLR1D genes. In the present case report, a patient with TCS with auricle dysplasia and hearing loss accompanied with intellectual disability is described. Sequence analysis was performed on blood samples from the patient and his father via oligonucleotide-based target capture, followed by next-generation sequencing. Alignment and variant calls were generated using the Burrows-Wheeler Aligner and Genome Analysis Toolkit, followed by bioinformatics analysis of the detected variants. A novel heterozygous mutation, c.911C > T (p.Ser304Leu), was detected in the TCOF1 gene, which was inherited from the father. The father of the patient only suffered from hearing loss. The present report is the first to identify an association between phenotypic variability and TCOF1 gene mutations and thus contributes to our understanding of the association between the genotype and phenotype in patients with TCS and offers clinically relevant information for diagnosis of the syndrome.
\end{abstract}

\section{Introduction}

Treacher Collins syndrome (TCS; OMIM no. 154500; omim. org/) is a well-described autosomal dominant type of mandibulofacial dysostosis (MFD), with an estimated prevalence of 1 in 50,000 live births (1). The earliest known reports of

Correspondence to: Dr Elena Papageorgiou, Access to Genome, Clinical Laboratory Genetics, Ethn. Antistaseos 33A, 55134 Thessaloniki, Greece

E-mail: elenpapage@hotmail.com

Key words: Treacher Collins syndrome, treacle ribosome biogenesis factor 1, point mutation, P53 the condition came from Thomson in 1849 (2) and Berry in 1889 (3), but the condition is officially named after E. Treacher Collins, who described the diagnostic criteria of this disease in 1900 (4). A more detailed description of the syndrome was provided by Franceshetti and Klein in 1949, who used the term MFD, and subsequently TCS was also referred to as Franceshetti-Klein syndrome (4).

TCS is characterized by symmetrical malformations with variable clinical features. Some typical characteristics of the disease are down-slanting palpebral fissures, hypoplastic zygomatic bone, lower eyelid coloboma associated with loss of the medial eyelashes, micrognathia, ear aplasia or microtia, conductive hearing loss and cleft palate (4). Although the disorder is thought to be of complete penetrance, clinical evaluation of affected patients showed incomplete penetrance (5) There is a high degree of intra- and interfamilial clinical heterogeneity, from severe craniofacial malformation to patients who are so mildly affected that they escape diagnosis (6); however, if such patients undergo more careful examination, minor malformations are often identified (6).

TCS exhibits genetic heterogeneity as well. To date, three genes have been identified to be associated with this disease: The treacle ribosome biogenesis factor 1 (TCOF1) gene on chromosome 5q32-33.1; the RNA polymerase I and III subunit D (POLR1D) gene on chromosome 13q12.2; and the RNA polymerase I and III subunit C (POLR1C) gene on chromosome $6 \mathrm{p} 21$, whereas the syndrome is inherited in a dominant manner through heterozygous mutations in the TCOF1 and POLR1D genes, mutations in POLR1C gene are inherited in an autosomal recessive manner and compound heterozygosity is required to be phenotypically classed as having TCS (7). TCOF1 mutations are the most common cause of TCS, with an estimated $60 \%$ of TCS cases attributed to de novo mutations of this gene $(6,7)$. The TCOF1 gene encodes the treacle protein, which is a nucleolar phosphoprotein that is trafficked between the nucleolus and cytoplasm; it is involved in rRNA and rDNA gene transcription affecting the proliferation and differentiation of neural crest cells during early embryogenesis (8). In total, $>150$ different mutations of TCOF1 associated with TCS, have been reported, proposed mutational hot spots on the gene indicate that exons 23 and 24 are responsible for 
$>30 \%$ of pathogenic mutations (9). In the majority of cases, the mutations are frameshift deletions or duplications leading to premature termination codons or mutations in the promoter region $(10,11)$.

The present study reports on a patient who presented with clinical features indicative of TCS with a heterozygous mutation in the TCOF1 gene. The mutation has not been described in the literature before, to the best of our knowledge. Parental clinical evaluation and testing were performed, and revealed that the mutation was inherited. The aim of the present study was to contribute to the understanding of the genotype-phenotype association in patients with TCS, and to offer clinically relevant information regarding the syndrome due to the high degree of intra- and inter-familial phenotypic variability.

\section{Case report}

A 3-year-old boy born to an unrelated healthy mother and a father who presented with hearing problems only, was admitted to Achepa Hospital for Special Needs (Thessaloniki, Greece) in January 2017 due to phenotypic dysmorphisms. Examination of the 3 year old boy revealed external and right middle ear dysplasia, low-set and cupped ears, almond-shaped eyes and down-slanting palpebral fissures (Fig. 1). The patient also had a permanent open bite and high arched palate, with mouth breathing and tongue thrusting. Low birth weight, due to feeding problems and micrognathia, and single transverse palmar crease were present. The patient had normal heart function with no heart abnormalities and exhibited mild intellectual disability (Table I). Based on the phenotypic features, a possible TCS diagnosis was made and genetic testing was recommended for the proband and his parents.

Genomic DNA from blood samples taken from the father and the proband was extracted using the QIAamp DNA Blood Mini kit (Qiagen,Inc.) according to the manufacturer's protocol. Regarding the specific gene mutation in the present case, PCR was performed for each sample using Qiagen HotStarTaq DNA polymerase was used with the following thermocycling conditions: $95^{\circ} \mathrm{C}$ for $15 \mathrm{~min}$; followed by 30 cycles of $94^{\circ} \mathrm{C}$ for $30 \mathrm{sec}, 58^{\circ} \mathrm{C}$ for $60 \mathrm{sec}$ and $72^{\circ} \mathrm{C}$ for $1 \mathrm{~min}$; with a final extension step of $72^{\circ} \mathrm{C}$ for $10 \mathrm{~min}$. The sequences of the primers were: TCOF_8 forward, 5'-TAAGGCCTCTGGACTTTATC-3' and reverse, 3'-CACAGTGAGAGGGGAGTAAG-5'. The coding regions and the intronic flanking regions $(+/-8 \mathrm{bp})$ of the TCOF1 (OMIM no. 606847; chr5) gene were completely sequenced (reference sequence, NM_001135243.1; ncbi.nlm. nih.gov). Sequence analysis was performed using oligonucleotide-based target capture (QXT; Agilent Technologies, Inc.) followed by next-generation sequencing (MiSeq; Illumina, Inc.) using M13 sequencing primers (Thermo Fisher Scientific, Inc.). Alignment and variant calls were generated using the Burrows-Wheeler Aligner and Genome Analysis Toolkit version 3.6 (software.broadinstitute.org/gatk/) followed by analysis of the predicted effects of the detected variants using ALIGN-GVGD version 2007 (agvgd.hci.utah.edu/) and SIFT version 6.1 (sift.bii.a-star.edu.sg/). Where necessary, Sanger sequencing was used to provide data for bases with insufficient coverage (mean coverage $<100 \mathrm{X}$ and/or minimum coverage of $<20 \mathrm{X})$. The possibility that pseudogene sequences or highly-homologous sequences may have interfered with the

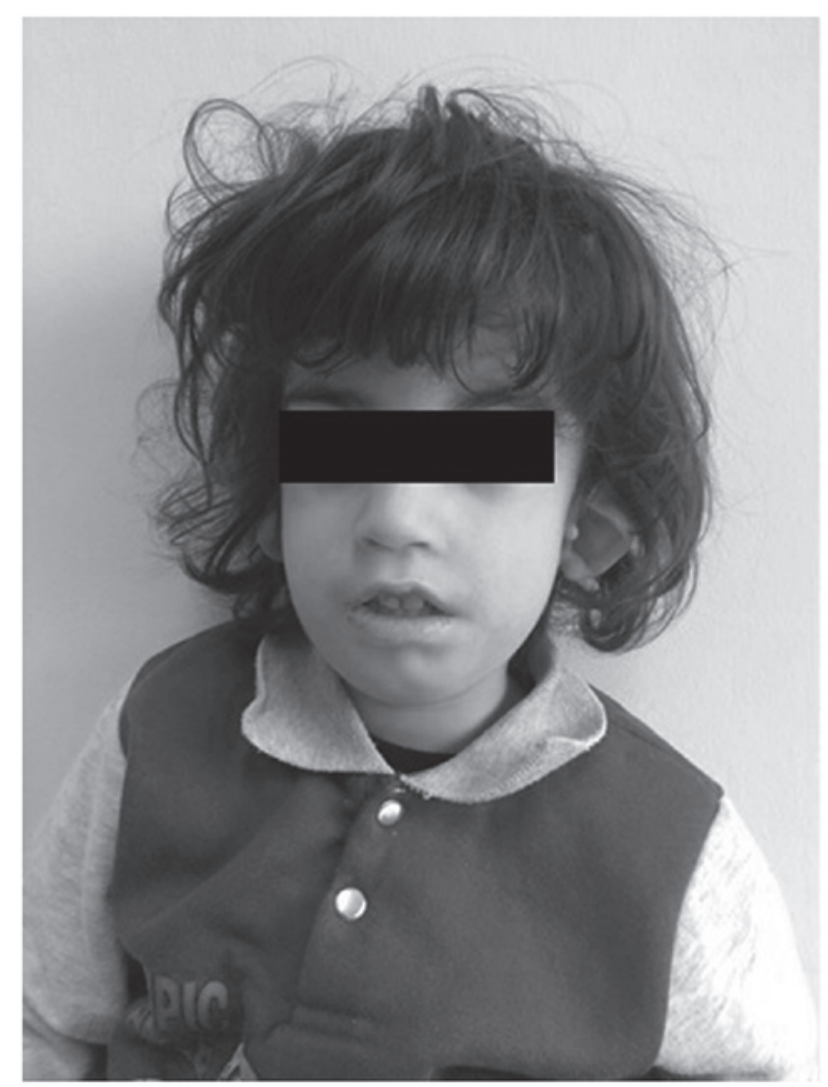

Figure 1. Photograph of the 3-year-old patient.

technical ability to identify the variants present in the analysis was not be excluded. All clinically significant and novel variants were confirmed by independent Sanger sequencing.

\section{Results and Discussion}

The c.911C > T (p.Ser304Leu) variant was detected in heterozygosity in the TCOF1 gene in both the patient and his father (Figs. 2 and 3).

In the present study, a patient with TCS and his father, who only has hearing problems, are described. The patient was determined to possess a previously unidentified mutation in the TCOF1 gene which was inherited from his father. It has been suggested that there may be hot spot mutation sites in exons 10, 15, 16, 23 and 24 of TCOF1 (12), but mutations have been identified throughout the coding region (13). The current patient inherited a mutation in exon 8 of TCOF1. Therefore, it is suggested that mutational analysis of the entire TCOF1 gene should be performed if a patient is suspected of having TCS.

There is no correlation between phenotypic variability and the location of the mutations within TCOF1 (14). In the present study the patient had a more severe phenotype than his father, despite them both having the same genetic variant in TCOF1 [c.911C > T (p.Ser304Leu)]. This may be due to the association of the treacle protein with P53. Treacle, the protein encoded for by TCOF1, is shown to have a role in regulating transcription of rDNA and in pre-processing the rRNA transcript (15). Inhibiting these functions, particularly in neural crest cells, results in apoptosis of the cells (16). However, P53 partial knockout in $\mathrm{TCOF}^{+/-}$mice is protective against the TCS phenotype (17). 
Table I. Clinical features of the patient and the father.

\begin{tabular}{|c|c|c|c|c|c|}
\hline Clinical features of the patient & Yes & No & Clinical features of patient's father & Yes & No \\
\hline Downward-slanting palpebral fissures & $\bullet$ & & Downward-slanting palpebral fissures & & - \\
\hline Zygomatic complex hypoplasia & & $\bullet$ & Zygomatic complex hypoplasia & & $\bullet$ \\
\hline Conductive deafness & $\bullet$ & & Conductive deafness & & $\bullet$ \\
\hline Mandibular hypoplasia & $\bullet$ & & Mandibular hypoplasia & & • \\
\hline Atresia of external ear canal & $\bullet$ & & Atresia of external ear canal & & • \\
\hline Microtia & $\bullet$ & & Microtia & $\bullet$ & \\
\hline Lower eyelid coloboma & $\bullet$ & & Lower eyelid coloboma & & - \\
\hline Cleft palate & $\bullet$ & & Cleft palate & & \\
\hline Cardiac malformation & & • & Cardiac malformation & & $\bullet$ \\
\hline Gastrostomy in the neonatal period & & • & Gastrostomy in the neonatal period & $\bullet$ & \\
\hline Delayed motor development & $\bullet$ & & Delayed motor development & & $\bullet$ \\
\hline Delayed speech development & $\bullet$ & & Delayed speech development & & - \\
\hline
\end{tabular}

\begin{tabular}{llcl}
\hline Ala & Ala & Ser & Ala \\
Ala & Ala & Serneu & Ala \\
& & & \\
\hline \hline
\end{tabular}
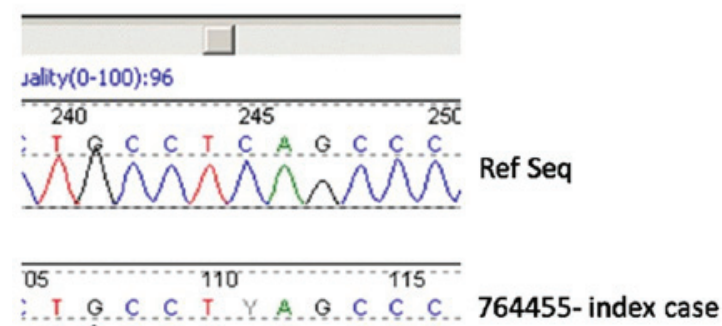
$M \wedge \wedge \triangle A \triangle \triangle \triangle \wedge A$

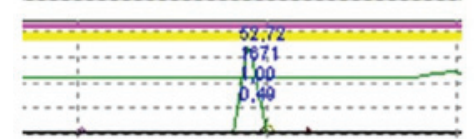

\begin{tabular}{llcl}
\hline Ala & Ala & Ser & Ala \\
Ala & Ala & SerReu & Ala \\
& & & \\
\hline \hline
\end{tabular}

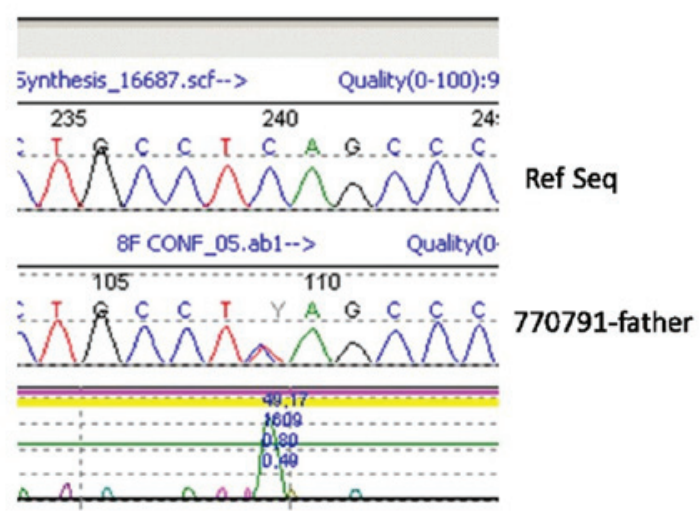

Figure 2. Sequencing results showing the mutation [c.911C >T (p.Ser304Leu)] found in the proband and his father. Ref seq, reference sequence.

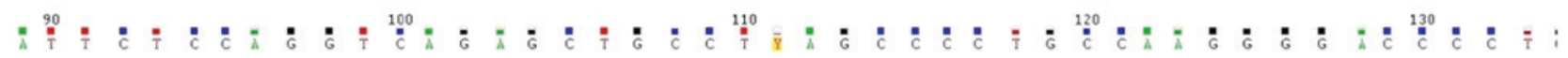

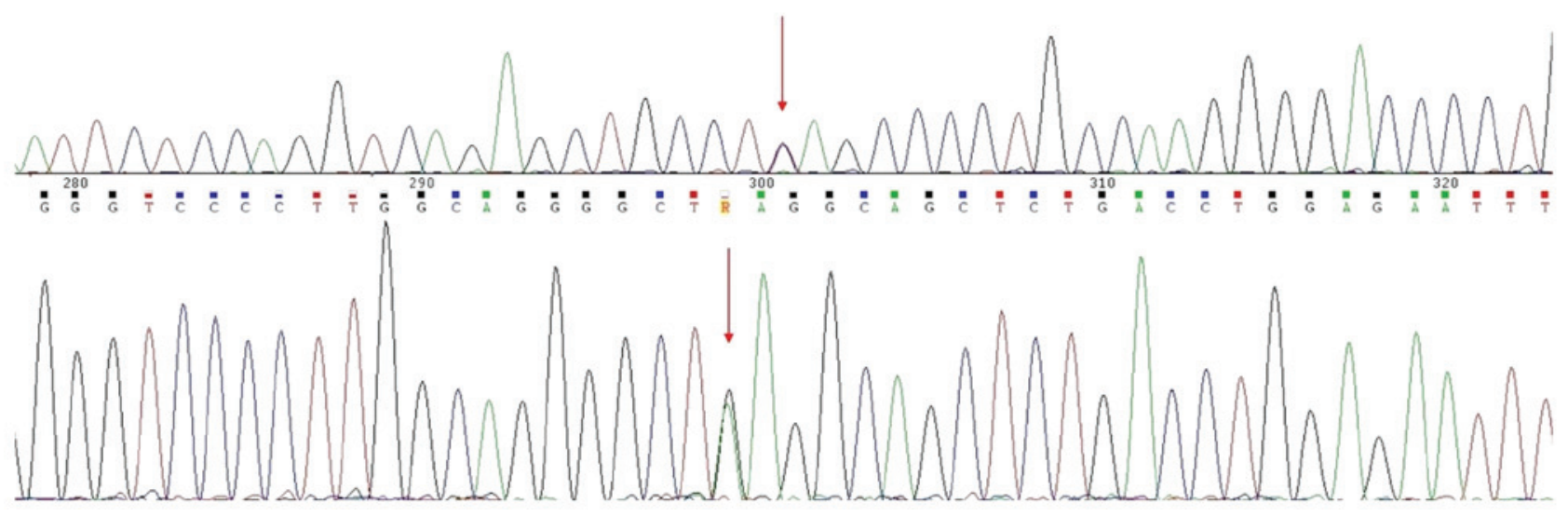

Figure 3. Point mutation [c.911C $>$ T (p.Ser304Leu)] verified by Sanger sequencing. Top, forward sequence; Bottom, reverse sequence. 
Neural crest cell development can be divided into three distinct stages: Formation, migration and differentiation (18). These cells originate from different positions along the anterior-posterior axis and they develop into various tissues. These regions of neural crest cells can be divided into four functional domains; cranial neural crest, trunk neural crest, vagal and sacral neural crest, and cardiac neural crest (19). Cranial neural crest cells give rise to distinct cell and tissue types but are generated transiently (19). Therefore, it is critical that the embryo generates and maintains a sufficient pool of neural crest progenitors that survive, proliferate, migrate and differentiate appropriately (18). Cell lineage tracing performed in E8.5 wild-type and $\mathrm{TCOF} 1^{+/}$mouse embryos revealed that there was no migratory nor pathfinding defects in cranial neural crest cell migration (20). This observation therefore indicated that TCOF1 does not play a role in neural crest cell migration and, furthermore, that aberrant neural crest cell migration is not the underlying cause of TCS. However, $25 \%$ fewer migrating neural crest cells were reproducibly observed in TCS embryos compared to their wild-type mice littermates, in a previous study (19). The deficiency of neural crest cells arises due to extensive neuroepithelial apoptosis from E8.0 to 10.5, which diminishes the neural stem cell pool from which neural crest cells are derived. Therefore, TCOF1 serves a critical role in neural crest cell formation and is required for neuroepithelial survival and neural crest cell proliferation (20).

The primary aim of the present study was to demonstrate the clinical heterogeneity of TCS syndrome and provide phenotypic evidence of affected humans. The c.911C $>\mathrm{T}$ (p.Ser304Leu) variant, detected in heterozygosity in the TCOF1 gene, has not been previously described in the literature, to the best of our knowledge. This variant however is reported in dbSNP (rs144193760; minor allele frequency, N/A; ncbi.nlm.nih.gov/snp/), ESP (minor allele frequency, $0.03 \%$; sp.gs.washington.edu/drupal/) and gnomAD (minor allele frequency, $0.018 \%, 50$ heterozygous individuals reported; gnomad.broadinstitute.org/); it affects a weakly conserved nucleotide [phyloP:0.44(-20.0;10.0)] and the predicted effects were inconclusive regarding its pathogenicity; $\mathrm{C} 15(\mathrm{GV}$, 73.35-GD, 97.78); deleterious (score, 0.04; median, 3.36). Thus, this mutation should be considered a variant of unknown clinical significance. Additionally, when the syndrome is caused by TCOF1 mutations, it is inherited in an autosomal dominant manner and significant inter- and intrafamilial clinical variability is common in TCS. As the present analysis does not exclude variants outside of the analyzed regions or variants which were not detected by the methodology used, the present study states that the current result does not confirm, but also does not exclude, that the clinical diagnosis of TCS was caused by mutation of the TCOF1 gene, and a more extensive analysis of the patients genome would be required to support or discredit the gene and the specific mutations involvement.

In conclusion, a patient with TCS and an unreported mutation within the TCOF1 gene [c.911C>T (p.Ser304Leu)] is described. This mutant gene was inherited from the father who only had hearing problems. The intrafamilial phenotypic variability, which was also observed in this case study, supports the hypothesis that genetic modifying factors may serve a regulatory role in the development of this syndrome.

\section{Acknowledgements}

Not applicable.

\section{Funding}

No funding was received.

\section{Availability of data and materials}

All data generated or analyzed during this study are included in this published article.

\section{Authors' contributions}

ElP was the major contributor in writing the manuscript. ElP, IP and EM analyzed the blood samples and found the familial mutation. AZ and EvP analyzed the data, performed the bibliographic research and drafted the manuscript. SF performed the clinical examination of the patient and his family. All authors have read and approved the final version of the manuscript.

\section{Ethics approval and consent to participate}

Informed consent for participation was obtained from the parents.

\section{Patient consent for publication}

Informed consent for publication was obtained from the parents.

\section{Competing interests}

The authors declare that they have no competing interests.

\section{References}

1. Caluseriu O, Lowry BR, McLeod R, Lamont R, Parboosingh JS, Bernier FP and Innes AM: The hutterite variant of treacher collins syndrome: A 28-year-old story solved. Am J Med Genet 161A: 2855-2859, 2013.

2. Thomson A: Notice of several cases of malformation of the external ear, together with experiments on the state of hearing in such persons. Mon J Med Sci 1: 420-425, 1846.

3. Berry GA: Note on a congenital defect (?coloboma) of the lower lid. R Lond Ophthal Hosp Rep 12: 255-257, 1889.

4. Dixon MJ, Treacher collins syndrome. Hum Mol Genet 5: 1391-1393, 1996.

5. Dixon MJ, Marres HA, Edwards SJ, Dixon J and Cremers CW: Treacher Collins syndrome: Correlation between clinical and genetic linkage studies. Clin Dysmorphol 3: 96-103, 1994.

6. Dixon MJ, Read AP, Donnai D, Colley A, Dixon J and Williamson R: The gene for Treacher Collins syndrome maps to the long arm of chromosome 5. Am J Hum Genet 49: 17-22, 1991.

7. Katsanis SH and Jabs EW: Treacher Collins Syndrome. 2004 Jul 20 (updated 2012 Aug 30). Pagon RA, Adam MP, Ardinger HH, Wallace SE, Amemiya A, Bean LJ,Bird TD, Ledbetter N, Mefford HC, Smith RJ and Stephens K (eds): GeneReviews ${ }^{\circledR}$ (Internet). University of Washington, Seattle, WA, 1993-2017.

8. Gonzales B, Henning D, So RB, Dixon J, Dixon MJ and Valdez BC: The Treacher Collins syndrome (TCOF1) gene product is involved in pre-rRNA methylation. Hum Mol Genet 14: 2035-2043, 2005.

9. Splendore A, Jabs EW, Félix TM and Passos-Bueno MR: Parental origin of mutations in sporadic cases of Treacher Collins syndrome. Eur J Hum Genet 11: 718-722, 2003. 
10. Splendore A, Fanganiello RD, Masotti C, Morganti LS and Passos-Bueno MR: TCOF1 mutation database: Novel mutation in the alternatively spliced exon $6 \mathrm{~A}$ and update in mutation nomenclature. Hum Mutat 25: 429-434, 2005.

11. Masotti C, Armelin-Correa LM, Splendore A, Lin CJ, Barbosa A, Sogayar MC and Passos-Bueno MR: A functional SNP in the promoter region of TCOF1 is associated with reduced gene expression and YY1 DNA-protein interaction. Gene 359: 44-52, 2005.

12. Splendore A, Silva EO, Alonso LG, Richieri-Costa A, Alonso N Rosa A, Carakushanky G, Cavalcanti DP, Brunoni D and Passos-Bueno MR: High mutation detection rate in TCOF1 among Treacher Collins syndrome patients reveals clustering of mutations and 16 novel pathogenic changes. Hum Mutat 16: 315-322, 2000

13. Wise CA, Chiang LC, Paznekas WA, Sharma M, Musy MM, Ashley JA, Lovett M and Jabs EW: TCOF1 gene encodes a putative nucleolar phosphoprotein that exhibits mutations in Treacher Collins syndrome throughout its coding region. Proc Natl Acad Sci USA 94: 3110-3115, 1997.

14. TeberOA, Gillessen-Kaesbach G, Fischer S, Böhringer S, AlbrechtB, Albert A, Arslan-Kirchner M, Haan E, Hagedorn-Greiwe M, Hammans $C$, et al: Genotyping in 46 patients with tentative diagnosis of Treacher Collins syndrome revealed unexpected phenotypic variation. Eur J Hum Genet 12: 879-890, 2004.
15. Hayano T, Yanagida M, Yamauchi Y, Shinkawa T, Isobe T and Takahashi N: Proteomic analysis of human Nop56p-associated pre-ribosomal ribonucleoprotein complexes. Possible link between Nop56p and the nucleolar protein treacle responsible for Treacher Collins syndrome. J Biol Chem 278: 34309-34319, 2003.

16. Trainor PA, Dixon J and Dixon MJ: Treacher Collins syndrome: Etiology, pathogenesis and prevention. Eur J Hum Genet 17: 275-283, 2009.

17. Jones NC, Lynn ML, Gaudenz K, Sakai D, Aoto K, Rey JP, Glynn EF, Ellington L, Du C, Dixon J, et al: Prevention of the neurocristopathy Treacher Collins syndrome through inhibition of p53 function. Nat Med 14: 125-133, 2008.

18. Trainor PA: Craniofacial birth defects: The role of neural crest cells in the etiology and pathogenesis of Treacher Collins syndrome and the potential for prevention. Am J Med Genet A 152A: 2984-2994, 2010.

19. Huang $X$ and Saint-Jeannet JP: Induction of the neural crest and the opportunities of life on the edge. Dev Biol 275: 1-11, 2004.

20. Dixon J, Jones NC, Sandell LL, Jayasinghe SM, Crane J, Rey JP, Dixon MJ and Trainor PA: Tcof $1 /$ Treacle is required for neural crest cell formation and proliferation deficiencies that cause craniofacial abnormalities. Proc Natl Acad Sci USA 103: 13403-13408, 2006. 